\title{
The precision of spectroscopic luminosity in A-F type stars and the classification of Am stars
}

\author{
C. Jaschek and A. Valbousquet \\ Observatoire de Strasbourg, URA 1280 CNRS, 11 rue de l'Université, 67000 Strasbourg, France
}

Received January 6; accepted February 27, 1997

\begin{abstract}
We analyze the work of three teams of classifiers providing spectral classifications in the range of late B-type to early F-type stars. With the help of a statistical model we derive an accuracy of \pm 0.7 luminosity classes for the spectroscopic luminosity class provided by classifiers. This is sligthly better than one luminosity class, and corresponds, in the region of the A-type stars, to a precision of about \pm 0.4 (absolute) magnitudes. We do not find differences in the precision of three series analyzed.

We analyze also the case of Am stars and conclude that the probability that two independent classifiers agree that a given object is $\mathrm{Am}$, is of the order of $60 \%$.
\end{abstract}

Key words: stars: distances — stars: fundamental parameters - stars: HR diagram

\section{Introduction}

The availability of a large quantity of new and precise trigonometric parallaxes from the HIPPARCOS satellite raises again the need for spectral classifications. This is due essentially to two different reasons.

In the first place because most of the program stars for the HIPPARCOS programs were selected on the basis of spectroscopic criteria (Am, Ap, delta Del stars, giants, subgiants, etc). Poor classifications produce biased samples, and biased samples produce biased average absolute magnitudes for a group.

In the second place, when calibrating the HR diagram, one would like to know the spectroscopic luminosity class of the objects being used in the sample, to have an external check upon the stars one includes.

The present paper is written with these two purposes in mind. In the first part we shall examine how well luminosity classes derived by different classifiers agree among them, and what can be said about the general reliability

Send offprint requests to: C. Jaschek, 37 Teso de la Feria 37008 Salamanca, Spain. of a spectroscopic luminosity class. Since our interest lies with early type stars, we shall examine this problem with samples of A-F type stars.

In the second part we shall examine one spectroscopically selected group of objects - the Am stars - to find out how well they are singled out by different classifiers, and how the classifiers agree among themselves.

\section{Comparison of luminosity classes}

In the A-F star region we have at present three different surveys, carried out on photographic plate material, obtained with slit spectrographs and classified in the traditional way in the MK system. These are

a) Cowley et al. (1969). The survey covers the B8-F0 stars, in the northern sky down to 6.5 and -10 declination. This survey shall be abbreviated CJ.

b) Garrison \& Gray (Gray \& Garrison 1987, 1989a, 1989b and Garrison \& Gray 1994). The survey covers most B8F2 stars, in both hemispheres at least up to 5.0 It shall be abbreviated as GG.

c) Abt \& Morell (1995) The survey covers most of the northern B8-F1 star, down to 6.5 and -20 declination. It shall be abbreviated as AbM.

We have provided just the rough limits of each survey, but for the strict definition of each program the reader should consult the original papers. It is clear from the preceding summary that a large superposition between the three surveys exists. The surveys have been carried out with different dispersions, namely 125 (CJ), 67 (GG) and $40(\mathrm{AbM}) \mathrm{A} / \mathrm{mm}$. All observers used the framework of the MK system and their classifications should thus have a high degree of homogeneity and of intercomparability.

\section{Samples of normal stars}

We can have a general idea about the trend by comparing the luminosity classes of the stars in common to the different surveys. We start by setting up a sample of stars classified simultaneously by all three teams. This sample has the disadvantage of being rather small, but on the 
other side the advantage that one is discussing the same objects. The numbers are given in Table 1, where also Am and Ap stars were included. It is obvious that AbM and CJ agree rather well, whereas GG show a smaller number of dwarfs and a larger number of intermediate luminosity classes, In fact if classes V, IV-V and IV are grouped together one finds a rather similar number of stars in all three teams. This implies that luminosity class IV presents problems. Since Table 1 contains only stars in common to the three samples, its spectral range is essentially reduced to A-type stars. In order to enlarge the coverage of spectral types and to increase the sample size we shall next examine the samples by comparing in one step stars common to GG and AbM and in another, stars common to AbM and CJ. We start with a comparison of GG and AbM samples. This is done in Table 2. A look at the table shows that the situation with respect to intermediate classes and to class IV is the same as in Table 1, as it should be. In order to examine more in detail where the discrepancies lye, we can break up the statistics into three subsets - early A type, middle and late A type, and early F type. Since the numbers are small we do not provide the figures resulting from the breakdown, but notice simply that in general, the sum of the stars classified by GG as V, IV-V and IV is about equal to the stars classed $\mathrm{V}$ and IV by $\mathrm{AbM}$ - in all three subsets.

Table 1. Comparison ot the luminosity classes of all stars in common to the three samples

\begin{tabular}{lccc}
\hline L. Class & GG & AbM & CJ \\
\hline V & 116 & 171 & 168 \\
IV-V & 27 & 1 & 6 \\
IV & 69 & 55 & 52 \\
III-IV & 8 & 2 & 2 \\
III & 25 & 22 & 37 \\
II-III & & & 1 \\
II & 2 & 1 & 2 \\
II-Ib & 2 & & \\
Ib & 1 & 2 & 2 \\
Ia & 3 & 3 & 3 \\
Am & 50 & 38 & 24 \\
p & 4 & 12 & 10 \\
\hline Total 307 & & & \\
\hline
\end{tabular}

We shall next examine the agreements or disagreements in classifying a given object. This is done in a series of breakdowns by luminosity class. We shall use only classes V, IV and III, because for the other groups the numbers are too small to be meaningful. Table 3 provides the breakdown. The result is rather surprising, in the sense that the coincidences in luminosity class are less than $50 \%$ in the best case.

Similar statistics can be obtained from a comparison of the stars in common to the AbM and CJ samples. Here
Table 2. Comparison of luminosity classes for the stars in common in the GG and AbM samples, including Am stars

\begin{tabular}{lcc}
\hline L. Class & GG & AbM \\
\hline V & 176 & 260 \\
IV-V & 45 & 1 \\
IV & 104 & 99 \\
IV-III & 15 & 6 \\
III & 41 & 44 \\
III-II & 1 & \\
II & 7 & 3 \\
II-Ib & 3 & - \\
Ib & 3 & 3 \\
Iab & & 2 \\
Ia & 6 & 5 \\
Am & 72 & 50 \\
\hline Total & 473 & 473 \\
\hline
\end{tabular}

Table 3. Stars classed by GG and AbM

\begin{tabular}{llllll}
\hline Class V. & & & & \\
& GG: & & V & not V & \\
& AbM & V & $42 \%$ & $43 \%$ & \\
Class IV & & not V & $15 \%$ & & Total number 295 \\
& GG: & & IV & not IV & \\
& AbM & IV & $19 \%$ & $42 \%$ & \\
Class III & & not IV & $38 \%$ & & Total number 148 \\
& GG: & & & & \\
& AbM & III & III & not III & \\
& & not III & $40 \%$ & $44 \%$ & \\
& & & & & \\
\end{tabular}

the comparison was done on the stars with HR numbers less than 3000, in order to have a similarly sized sample to the first one. Table 4 provides the overall statistics, and Table 5 the breakdown in luminosity classes.

A comparison of Tables 3 and 5 shows that in general the number of coincidences in luminosity class is large only for class $\mathrm{V}$, being of the order of $50 \%$. For luminosity classes IV and III the percentage of agreements between two classifiers is of the order of $17 \%$. Such low percentages of agreement may come as a shock to those who believe that the luminosity class attributed by a classifier is accurate and can be translated safely through a calibration into an accurate spectroscopic parallax.

We have to look now for an explanation of such low percentages. $\AA$ Assume that we compare the results of two classifiers A and B, both classifying the same stars and each of them having a classification error in luminosity class (LC) of sizes $E(\mathrm{~A})$ and $E(\mathrm{~B})$ respectively. Since the MK standards are given for entire luminosity classes (some exceptions like IV-V left aside for the moment), it seems that $E$ should be of the order of one luminosity class or less. If the error is of say 0.75 this has only a 
Table 4. Comparison of luminosity classes in the AbM and CJ samples, including Ap and Am stars

\begin{tabular}{lcc}
\hline L. Class & AM & CJ \\
V & 223 & 268 \\
IV-V & 5 & 3 \\
IV & 74 & 41 \\
III-IV & 5 & 3 \\
III & 41 & 28 \\
II & 1 & 2 \\
Ib & 3 & 2 \\
Iab & 1 & - \\
Ia & 4 & 5 \\
p & 30 & 30 \\
m & 50 & 55 \\
\hline Total & 437 & \\
\hline
\end{tabular}

Table 5. Stars classed by AbM and CJ

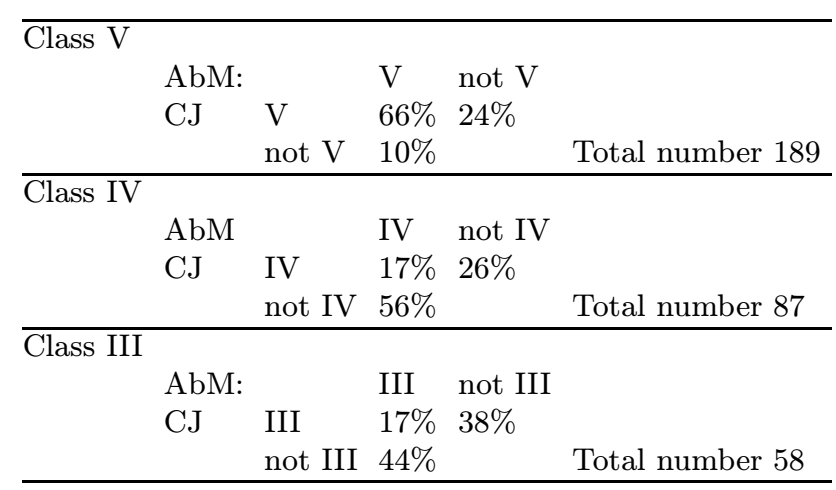

statistical sense, since the luminosity classes are only quoted in units (or half units if one considers IV- III to lay strictly midway between IV and III). Let us assume next that the errors are distributed following a Gaussian distribution. It is easy then to predict the expected differences in luminosity class. A complication arises because of the fact that not all luminosity classes are equally frequent in a magnitude limited sample. Because of this one must introduce the distribution of the relative frequency of the different luminosity classes. We have adapted the relative frequencies from the sample counts in $\mathrm{AbM}$, given in Table 6.

Table 6. Relative frequency of stars of different luminosity classes in the magnitude limited AM sample

\begin{tabular}{rr}
\hline Class V & 80 \\
IV & 40 \\
III & 20 \\
II & 10 \\
I & 5 \\
\hline
\end{tabular}

We can now combine the error distribution with the frequency distribution of the luminosity classes. Tables 7 , 8 and 9 provide the results for different errors. To make the statistics clearer and to avoid small numbers, we have simplified the results by quoting only two results: "Class V" and "not class V" and similarly for other luminosity groups. Since there are too few stars of luminosity classes II and I for good statistics, we have given only the results for classes V, IV and III.

Table 7. Incidence of the classification errors in comparisons of the luminosity classifications of two classifiers A and B. $E(\mathrm{~A})=E(\mathrm{~B})=1.0 \mathrm{LC}$ (Luminosity classes)

\begin{tabular}{rrrll}
\hline \multicolumn{1}{l}{ Class V } & \multicolumn{3}{l}{ Class IV } & Class III \\
\hline a) Luminosity class V & & & \\
Obs. A: & & V & not V \\
Obs. B: & V & 45 & 27 \\
& not V & 27 & \\
\hline b) Luminosity class IV & & & \\
Obs. A: & & IV & not IV \\
Obs. B: & IV & 17 & 41 \\
& not IV & 41 & \\
\hline c) Luminosity class III & & & \\
Obs. A: & & III & not III \\
Obs. B: & III & 29 & 35 \\
& not III & 35 & \\
\hline
\end{tabular}

Table 8. Same as Table 7 but for $E(A)=1.0 B=0.5$ L.C.

\begin{tabular}{lllll}
\hline a) Luminosity class V & & & & \\
& Obs. A: & & V & not V \\
& Obs. B: & V & 52 & 22 \\
& & not V & 25 \\
\hline b) Luminosity class IV & & & & \\
& Obs. A: & & IV & not IV \\
& Obs. B: & IV & 17 & 35 \\
& & not IV & 47 & \\
\hline c) Luminosity class III Obs. A: & & III & not III \\
& Obs. B: & III & 18 & 37 \\
& & not III & 44 & \\
\hline
\end{tabular}

In order to save space, the tables have been compressed, so that each table contains three small tables, referring respectively to classes V, IV and III. Each of them provides the percentage of classifications predicted by the two classifiers. For an illustration, let us take case a) (luminosity class V). The table provides the percentage of how many stars were called by the two classifiers as being class $\mathrm{V}$ and how many were not called class $\mathrm{V}$ by one or the other classifier. 
Table 9. The same as table 7 but for $E(\mathrm{~A})=1.0 B=1.5$ L.C.

\begin{tabular}{rlll}
\hline a) Luminosity class V & & & \\
Obs. A: & & V & not V \\
Obs. B: & V & 41 & 29 \\
& not V & 30 & \\
\hline b) Luminosity class IV & & & \\
Obs. A: & & IV & not IV \\
Obs. B: & IV & 14 & 49 \\
& not IV & 37 & \\
\hline c) Luminosity class III & & & \\
Obs. A: & & III & not III \\
Obs. B: & III & 11 & 44 \\
& not III & 44 & \\
\hline
\end{tabular}

Note: All figures given in Tables 7, 8 and 9 are percentages based upon samples of 10000 stars.

By examining the tables, one can appreciate that the coincidence of the classifiers should be largest in class $\mathrm{V}$ $(50-40 \%)$, whereas in other luminosity classes the coincidence should be smaller $(30-11 \%)$. The percentages vary as a function of the relative errors of the two classifiers, but it is clear that the different values of $E(B)$ does not change the basic fact that the percentage of coincidences in classes other than $\mathrm{V}$ is small.

We can compare next the results of the real classifications with Tables 7, 8 and 9 which provide the theoretical results. One sees immediately that the real counts agree rather well with the predictions of Table 7 , where equal sized errors of both classifiers were assumed. Since our samples are small, in a statistical sense, it is unrealistic to try to find the differences between classifiers. We had assumed in Table 7 that $E(\mathrm{~A})=E(\mathrm{~B})=+1.0$ L.C. and this is perhaps slightly too large. According to Houk, as quoted in Jaschek \& Jaschek (1987), the dispersion is +0.8 luminosity classes (LC). We have verified this by means of an analysis of the dispersions of the three series (AbM, GG and CJ) for stars in common. We have obtained an average dispersion of $+0.7 \mathrm{LC}$ Next we have redone the analysis of Table 7 with $E(\mathrm{~A})=E(\mathrm{~B})=0.8$ and 0.6 L.C. We shall not quote the values, but simply the results. The coincidence is best between +0.6 and +0.8 . If we take +0.7 for the mean, we find thus the same value derived from the abovementioned analysis of the dispersions. We are thus left with a dispersion of +0.7 L.C., for the three spectral classification series. This implies that the spectroscopic luminosities are sligthly better than one luminosity class. Please observe that when two series are compared, the dispersion is +1.0 L.C. To translate this in terms of absolute magnitude in this part of the HR diagram, we have used Schmidt-Kaler's calibration (1982) of luminosity class as a function of absolute magnitude in the region B8-F2.
It can be seen that an indetermination of plus minus 0.7 luminosity classes corresponds to something between 0.2 and 0.5 magnitudes, for luminosity classes $\mathrm{V}$ to III. We adopt 0.4 as an average, which corresponds to a relative error of the parallaxes of $19 \%$. Since the errors of the HIPPARCOS parallaxes are 0"0015 (Arenou et al. 1995) all stars nearer than about 125 pc have a better distance determination from the Hipparcos parallax than from spectroscopic parallax. Only beyond this limit, spectroscopic parallaxes are better. It should however be stressed that this is only true if one can take care properly of the many secondary effects like binarity, rotation, metallicity, evolutionary status and interstellar reddening.

A study of the absolute magnitudes of the MK standards by means of the parallaxes from the HIPPARCOS mission is under way.

\section{Samples of Am type stars}

We analyze now the stars classified as Am. Let us recall that Am stars are objects for which one quotes generally three spectral types- one corresponding to the strength of the Ca II lines, one to the strength of the hydrogen lines and a third one to the strength of the metallic lines. This precept has been followed by GG and AbM, but not by CJ, who called a star simply Am, without assigning the three types. CJ classify however a number of stars as Am:, i.e. marginal Am. GG follow a similar practice in introducing the proto-Am stars, stars which are metallic lined, but to a lesser degree. AbM do not quote marginal or proto-Am stars, although from the publication it is easy to see that such specimens do exist - like for instance an object classified B 9.5-A0-A1. Since these refinements are difficult to disentangle in a statistics based upon small samples, we have decided to apply the uniform procedure to consider Am stars everything which is called Am, Am:, marginal or proto-Am by the different authors.

For the theoretical prevision, the case is simpler than the preceding cases, since an object is either Am or it is not Am. Assuming that the classifier A has $E(\mathrm{~A})=1$, the proportion of the coincidences should be $80 \%$ for $E(\mathrm{~B})=$ $0.5,70 \%$ for $E(\mathrm{~B})=1.0$ and $57 \%$ for $E(\mathrm{~B})=1.5$. Here $E(\mathrm{~A})$ and $E(\mathrm{~B})$ design in a wide sense the accuracy of classification. To assume $E(\mathrm{~A})=1=E(\mathrm{~B})$ means simply that both $\mathrm{A}$ and $\mathrm{B}$ are equally good classifiers. $E(\mathrm{~A})>$ $E(\mathrm{~B})$ means that $\mathrm{A}$ is better than $\mathrm{B}$ and so on.

We pass next to the real samples. From Table 1 it can be seen that GG had $50 \mathrm{Am}$ stars in the sample, whereas AbM had only 38 and CJ, 24. When analyzed in detail, GG finds more early type Am's than AbM. The detailed comparison is given in the first part of Table 10 .

A similar comparison can be made between AbM and CJ. The results are given in the second part of Table 10 .

In view of the small number of objects involved, we can only state that about $60 \% \pm 6 \%$ of the classifications coincide (close to the $70 \%$ prediction) and thus that the 
Table 10. Stars classed Am

\begin{tabular}{|c|c|c|c|}
\hline $\begin{array}{r}\text { 1) GG and AbM } \\
\text { GG: } \\
\text { AbM: }\end{array}$ & $\begin{array}{c}\text { Am } \\
\text { not Am }\end{array}$ & $\begin{array}{l}\text { Am not Am } \\
57 \% 14 \% \\
28 \%\end{array}$ & Total number 70 objects \\
\hline $\begin{array}{r}\text { 2) } \mathrm{AbM} \text { and CJ } \\
\text { AbM: } \\
\text { CJ: }\end{array}$ & $\begin{array}{c}\text { Am } \\
\text { not Am }\end{array}$ & $\begin{array}{l}\text { Am not Am } \\
62 \% 13 \% \\
13 \%\end{array}$ & Total number 68 \\
\hline
\end{tabular}

three classifiers have about the same precision. The difference of 14 and $28 \%$ of the first table of non-coincidences is at the limit of the statistical fluctuation, so that it cannot be used to decide which of the classifiers is better.

In what precedes we have neglected certain facts which also have an incidence upon the results- for instance that the MK standards used for classification are not exactly the same in all three samples, a fact which may lead to small differences. Such small differences are however difficult to pin point with (small) samples of a few hundred stars. Another fact is that the different plate dispersions used in the surveys could also have an influence upon the results. However since the standards used should be affected in a similar way than the objects being classified, it is hard to believe that this could introduce large differences.

Rotation is another factor which may introduce difficulties. GG and AbM have paid special attention to rotation. CJ did not pay special attention to rotation because the smaller dispersion used $-125 \mathrm{~A} / \mathrm{mm}$ - makes the classification less sensible to rotation, except for stars having $V \sin i$ larger than $200 \mathrm{~km} / \mathrm{s}$, which are rather infrequent in the range of A type stars.

On the other hand, it is gratifying to see that the results for both dwarfs and Am stars show that there are no big differences between classifiers, or in other words, that when the prescriptions of the MK system are followed, a similar accuracy is reached, regardless of the classifier. Although this contradicts what has been written sometimes about the existence of more precise clasifications (i.e. more precise than others), our conclusion is entirely reasonable within the framework of the MK classification.

\section{Summary}

We have reached in this paper three conclusions, namely a) that the average precision of a MK luminosity class given in the literature is \pm 0.7 L.C., which is equivalent to \pm 0.4 magnitudes in the spectral range of $\mathrm{A}$ to $\mathrm{F} 2$ stars.

b) That the probability that two observers agree to call an object a "metallic line star" is of $60 \%$.

c) A detailed comparison of three different series of classifications carried out in the framework of the MK system shows that in the three cases the precision is very similar, a fact which is in line with the expectations.

Acknowledgements. We thank the referee, Prof. B. Hauck for comments leading to the improvement of the manuscript. One of us (C.J.) is indebted to M. Golay for many useful discussions concerning Am stars.

\section{References}

Abt H.A., Morrell N.I., 1995, ApJS 99, 135

Arenou F., Lindegren L., Froeschlé M., Gomez A.E., Turon C., Perryman M.A.C., Wielen R., 1995, A\&A 304, 52

Cowley A.P., Cowley C., Jaschek M., Jaschek C., 1969, AJ 74, 373

Garrison R.F., Gray R.O., 1994, AJ 104, 1556

Gray R.O., Garrison R.F., 1987, ApJS 65, 581

Gray R.O., Garrison R.F., 1989a, ApJS 70, 263

Gray R.O., Garrison R.F., 1989b, AJ 69, 301

Jaschek C., Jaschek M., 1987, "The classification of stars". Cambridge Univ. Press, p. 40

Schmidt-Kaler Th., 1982, Landolt-Boernstein, Neue Serie, Bd. 2, Teilband 6, p. 1 